\title{
Problems and Countermeasures in the Network Construction of Private Hospital
}

\author{
Shuchen Liu \\ Southeast University, Nanjing, Jiangsu, China, 211189 \\ hunter2011@foxmail.com
} Keywords: Network construction, Civilian-run hospital private hospital, Problems, Countermeas-
ures.

\begin{abstract}
Network construction does not constitute a difficulty in public hospitals in China. But it is a headache for the civilian -run hospitals. Compared with the governmental hospital, the civilianrun hospital is in a disadvantageous position in finance, technology and talents. Due to the comprehensive disadvantages, the network construction in civilian-run hospital also falls behind that of governmental hospital. This paper analyzes the problems of network construction in private hospital and puts forward the corresponding measures in order to provide some references for the related researches.
\end{abstract}

\section{Concept of Private Hospital}

Civilian-run hospitals are the medical institutions that are funded by social capital and approved by the administrative department of public health. The biggest characteristic of civilian-run hospital is self-supporting, self-management, self-development and self-perfection. This kind of hospital belongs to private hospitals in foreign countries. At present, China's civilian-run hospitals are basically equivalent to private hospitals, so it is also known as private hospitals for civilian-run hospitals. China has made many policies to encourage and support social capital to enter the medical field, and promote the diversification of investment subjects in medical institutions, and provide the basic guarantee for the development of private hospitals at the policy level. But there are still many problems in private hospitals, such as: the degree of social credibility needs to be further improved, the medical personnel should be strengthened, the regulations need to be perfected, and the fund is generally insufficient. what's more, owing to the fact that the history of civilian-run hospitals is relatively short, specifically established after China's opening-up. There are not many large scale civilian-run hospitals which are Top-grade ones and most of civilian-run hospitals are Second or below second grade, thus information construction is less developed in these hospitals.

\section{Necessity of Network Construction of Private Hospital}

As the certain trend of the social development in the future, infor matization has already brought about some profound changes to many industries. It can also change the hospital a lot. with a development for quite a while, civilian-run hospitals need to be further improved. At the present, the management concerning basic stuff, finance, outpatient, medicine and logistics is relatively of the traditional type and some even fall behind with the times, in great needs of being upgraded, thus making the information construction necessary and important. The role is not only to solve the current problem of hospital survival, but also the basis of long-term development of the hospital. The informationization construction of private hospitals in China started late, and there is still a big gap in the construction of public information. Hospital network not only serves as a window for the community and patients to know about the hospital but also helps to improve the management of the hospital. Thus it can provide better service to the patients. Good hospital website can help to establish and improve the image of the hospital, strengthen the connection between the hospital and the community, and greatly improve the information level of hospital staff. With the development of the national economy, the size of the hospital continues to expand, to redesign a set of new, more powerful, scalable hospital network, better serve the vast number of patients, better publicity, im- 
prove the hospital's social recognition is very necessary. Therefore, the construction of private hospital network can improve the hospital's work efficiency, and has great significance to comprehensively promote the hospital information construction.

\section{Problems in the Network Construction of Private Hospital}

\section{Ideological Problem}

Ideological Problem mainly refers to less awareness on the part of the management. Currently, some administrators of the civilian-run hospitals fail to realize the positive impact brought by the establishment of an effective computer network, thinking it as a waste of money, thus being reluctant to bring in facilities. Other administrators follow suit blindly by simply introducing certain facilities without taking their own issues into consideration. Still other administrators simply skim the surface by installing certain facilities without taking them into full play. These facilities are not properly used, causing a waste in some sense. All of the above problems are caused by the insufficiency of understanding and lack of the awareness of the importance of network construction on the management's part, which poses a great danger to the operation and development of the civilianrun hospitals.

Talent and Financial Problem. Human power, capital, and material are the three decisive elements of development. First and foremost, lack of professional personnel is a biggest obstacle to the construction of informationalized network. Experts on computers rarely stay longer in the vivilianrun hospital because of it's inability to provide long-term employment guarantee. so most civilianrun hospitals fail to keep computer experts. Among the existing staff, retired staff account for a large percentage. Existing computer operators are graduates from technical training schools with low level informationalized skills. University graduates have less desire to work in the civilian-run hospitals.

Secondly, compared to public hospitals, a majority of private hospitals lack of funding and facilities. New purchase equipment due to excessive pursuit of low price, pay attention to short-term benefits, quality is low, and even a loss. Original professional technical level is uneven, medical equipment inspection accuracy and a discount, will inevitably lead to service projects, service, quality of service are subject to certain restrictions. It will restrict the development of the hospital. Private hospital information construction is still unsatisfactory. Reason is decided by the nature of private hospitals, it is funded and constructed by the investors of the hospital, investors are most concerned about is cast into the money if you can translate for more interests, and information on them is the icing on the cake. So, this has led to the construction of private hospital information is currently very poor. But information is the inevitable trend. Like mobile phones, more than a decade ago, the mobile phone is a luxury for us, and now is a necessity. Information is the same reason. It is now possible for private hospitals are a luxury, but the next few years, will inevitably become a necessity. And public hospitals are now aware of this, so public hospitals to invest in information construction, from millions to tens of millions of. The private hospitals always do not want to spend the money to construct the hospital network.

What has been discussed above have become the most realistic problems in the process of network construction.

Security Problem. Information security is one of the important themes in the Internet era. Network security has its own particularity. Not given enough care, it could cause the loss of money and even life. Even the smallest cost would be the leaking of confidential and the loss of information, thus giving rise to the inconveniences on the patients and malfunction on the part of the hospitals. In the network construction of hospitals (especially the private hospitals), we must attach great importance to this problem. Information security of some civilian-run hospitals is also an important factor in the network of hospitals. The weak part of the such hospitals is the door lock. If the key was accidentally lost, the weak electricity has been entered into by other people, the consequences of unintended consequences. Due to the current operation of the hospital relies on information system strengthens day by day, the resulting all kinds of medical data and information is to several 
times the speed continues to grow. However, from the point of view of investigation of hospital network construction, most hospitals lack corresponding computer professional talent of hospital network operation and maintenance management, which are often appear some network problems in network cannot be normal use. In addition, even some hospitals have the specialized network management personnel, also exists network security awareness is not strong, the important medical information. Information and data backup is not timely or even no backup, password setting simple, hospital network account leakage or lent to others and situation. The malicious attack is the biggest threat to the hospital computer network, and the adversary's attack and computer crime belong to this category. some operators even spend much time on playing on-line games on hospitals' computers. Such attacks can be divided into the following two: one is the active attack, it has chosen to destroy the effectiveness and integrity of the information in a variety of ways; the other is a passive attack, it is not affect the normal work of the network. To intercept, steal, decipher to obtain an important confidential letter urgent. These two attacks can cause great harm to the computer network, and lead to the leakage of confidential data. Hackers and computer virus to the enterprise network and the network security constitutes a great threat, every enterprise and network operators to spend a lot of manpower and material resources for the party and the network security, so to prevent manmade malicious attacks will be the focus of the work of hospital network security. It may become a shortcut to the computer virus and hacker intrusion hospital network system.

Maintenance Problem. As a special industry in the society, the private hospital has a direct relationship with people's health and the continuous improvement of the economic level of our country. The traditional management mode has been unable to meet the needs of modern hospital management, the traditional management mode is limited, and is easy to be influenced by environment and technology. Private hospital network system and hospital management and operation have become more and more closely related, once the hospital network failure, will give the whole hospital has adverse consequences, and even to the hospital and the patient cannot measure the loss. The stability of the private hospital network is very important, and the reliability and stability of the hospital network is the embodiment of the hospital's comprehensive ability, and it is also the condition of hospital management. Stable network is the basis for the work of the hospital. The main performance of the server in the lack of data backup processing, server work environment is poor, and there is a lack of server hardware maintenance of the phenomenon, the lack of daily cleaning of the server, the server is affected by the surrounding environment, the performance of the play a serious impact. The lack of internal network system management system is an important factor to affect server performance. Due to the lack of management system, some of the staff on the server to install a source of unknown sources of software, to the server's network security buried hidden trouble. Moreover, the lack of professional network system management personnel is also an important factor.

Weak Fundamental Infrastructure Problem. At the present, a large number of civilian-run hospitals are run with weak fundamental infrastructure. Some of such hospitals even transformed warehouses into wards. some civilian-run hospitals are suffering from the shortage of computer rooms which can meet the requirements of the network construction. some others don't install air conditioners . still other civilian-run hospitals can not guarantee 24 hours no-stop power supply. All of the above problems relating fundamental infrastructures make the network construction unable to be carried out from the very beginning. Under such circumstances, even the network has been constructed, it is unable to function.

\section{Countermeasures in the Network Construction of Private Hospital}

Improve the attention on network construction on the part of the management. Greater attention to the importance of network construction should be paid by the management. Because the management's attitude is a necessity to push forward the network construction. Therefore, it requires civilian-run hospitals of all kind to give priority to the establishment and development of 
network . It is also advisable for civilian-run hospitals to set up certain agents to guarantee the operation. Furthermore, publicity of the network should be strengthened, ensuring the use of network permeates to the every other part of their daily work .

Increase Talents and Capital Input. Generally speaking, civilian-run hospitals should be willing to invest in talents and capital resources. For the investment in talents, some hospitals can employ university graduates on computers, provide training courses for their staff, encouraging them to take computer exams, organize learning tour for hospital staff to learn more about network techniques. And they can re-employ the retired computer staff who once worked in the public hospitals. For a few hospitals which can not fix the problem of lacking computer expertise can outsource their needs to other specialized agents. For the investment in capitals, there are three ways to improve the capital investment. firstly, civilian-run hospitals should take the opportunity of the urgent need to develop on the government's part. Secondly, attract more funds from the society . Thirdly, these hospitals should fund it on their own, for example, setting up special fund for the construction of network, loaning from banks, collecting money from hospital staff, etc. which can improve the work efficiency greatly. For those weak in fundamental infrastructure, it is in great need to enhance the investment in effective facilities to make them qualified for the network construction.

Strengthen Security Protection. Network security management system is a major problem in the hospital network construction. Therefore, in order to further strengthen the management of hospital network, we need to establish a sound system of hospital network security management as soon as possible. Data management is the core of the entire network management, to strengthen the network data backup and encryption management, assign professionals to manage, and strict data use regulations, and effectively protect the hospital and patients. Virus in addition to the usual workstation anti-virus, email antivirus and gateway antivirus has become the key to eliminate the virus source. We should also be used to scan the scanner software, security checks, to find loopholes and timely repair, in order to prevent hacker attacks. The IOS ENABLE operating system is used to protect the security of the network layer. It can use UNIX system to ensure the security of the network layer. It can use UNIX system to ensure user identity, user authorization and security. The security file of /etc/hosts., such as equiv CISCO file, is used to prevent illegal users from using TELNET and FTP. Hospitals must ensure the realization of the safe network operation, which is a bottom line .

Enhance Network Maintenance. It is necessary and essential to keep the information system in a sustainable operation, therefore the management and maintenance of network become particularly important.Network maintenance of private hospitals, includes the software aspect and the hardware aspect. Software maintenance is related to the stability and reliability of the network system, in order to protect the network security, improve the network security of private hospitals, to deal with the hospital network security, set up access, the use of password, digital signature means to verify the identity of visitors, and often change password, improve the hospital network security. In order to ensure the stability of the whole system, maintenance must be sustained, and the occasional maintenance is not practical. On the other hand, the use of computer time is too long, will produce useless software and garbage, and with the software to install some software vulnerabilities may also be used by the virus, and therefore should be regularly uninstall some of the computer software, and upgrade commonly used. We should formulate relevant maintenance plan, regular maintenance of the entire network and software system. Hardware maintenance is also very important. Hospital network maintenance first of all to the hospital's UPS maintenance, to avoid the occurrence of accidents caused by network paralysis. Once the power failure, the hospital's a lot of information will not be saved, such as a number of information and registration fees, and registration information, it is possible because of the interruption and can not be saved, causing huge economic losses to the hospital, UPS maintenance should be equipped with UPS film, to protect the business in the event of a sufficient buffer time, complete information preservation. UPS equipment must be conducted a week to ensure that the UPS equipment is in normal condition. 


\section{Conclusion}

Civilian-run hospitals have taken up half of the hospitals nationwide, but their construction scale, service quality are much less developed than the public hospitals. The information construction poses a great challenge for the civilian-run hospitals. It is of great significance to intensify their network construction. Civilian-run hospital systems in network construction can greatly improve the operation efficiency of the hospital, and strengthen the comprehensive management ability of the hospital. The hospital network is the important basis for the construction of a modern hospital. Due to the lack of attention on the management part, the disadvantages of funds, technology and talents, the private hospitals have more problems in the construction of the network construction compared with public hospitals, such as financial problem, security problem and maintenance problem. The existence of these problems has seriously affected the process of network construction in private hospitals. This paper tries to take corresponding measures to strengthen the network construction in Civilian-run hospitals in order to promote the modernization process of private hospitals.

\section{References}

[1] J.M. Xu, Study on Problems and Countermeasures in the construction of hospital network, Electronic Test, 18(2014)113-115.

[2] C. Liu, Brief Analysis on Hospital Network Construction Existing Question and Countermeasure, Computer Knowledge and Technology (Academic Exchange), 12(2011)280-285

[3] F.H. Liang, J.W. Ke, L.L. Chen, W. Yang, X.J. Xue, Y. Shi, The Optimization Project of Separate and Combined Internal and External Network in Hospital Network Construction, Intelligent Building \& City Information, 7(2015)75-79.

[4] Y. Li, Research on the Development of Private Hospital under the Background of New Medical Reform in China, Tianjin University, 2012. 